\title{
Isatisine A, a Novel Alkaloid with an Unprecedented Skeleton from Leaves of Isatis indigotica
}

Ji-Feng Liu, Zhi-Yong Jiang, Rui-Rui Wang, Yong-Tang Zheng, Ji-Jun Chen*, Xue-Mei Zhang, Yun-Bao Ma

State Key Laboratory of Phytochemistry and Plant Resources in West China, Kunming Institute of Botany, Chinese Academy of Sciences, Kunming 650204, Yunnan,

P. R. China

Tel: $+86-8715223265 \quad$ Fax: $+86-8715223265$

E-mail: chenjj@mail.kib.ac.cn 


\section{Contents}

Page S 1

Page S 2

Page S 3

Page S 4

Page S 5

Page S 6

Page S 7

Page S 8

Page 59

Page S 10

Page S 11

Page S 12

Page S 13

Page S 14-16

Page S 17-18

Page S 19

Page S 20

Page S 21
The ${ }^{1} \mathrm{H}$ NMR spectrum of compound 1

The expanded ${ }^{1} \mathrm{H}$ NMR (7.0-8.0 ppm) spectrum of compound 1

The expanded ${ }^{1} \mathrm{H}$ NMR (3.0-5.0 ppm) spectrum of compound 1

The ${ }^{13} \mathrm{C}$ NMR spectrum of compound $\mathbf{1}$

The HSQC spectrum of compound $\mathbf{1}$

The expanded HSQC spectrum of compound 1

The HMBC spectrum of compound 1

The expanded (3.0-5.0 ppm) HMBC spectrum of compound 1

The expanded (7.0-9.0 ppm) HMBC spectrum of compound 1

The ${ }^{1} \mathrm{H}-{ }^{1} \mathrm{H}$ COSY spectrum of compound 1

The expanded ${ }^{1} \mathrm{H}-{ }^{1} \mathrm{H}$ COSY spectrum of compound 1

The IR (KBr) spectrum of compound 1

The FABMS of compound 1

The HRESIMS of compound 1

The ${ }^{1} \mathrm{H}$ NMR spectrum of compound 2

The FABMS of compound 2

The HPLC experiment of compound 1 and 2

The X-ray diffraction of compound 1 
The ${ }^{1} \mathrm{H}$ NMR spectrum of compound 1

jd18a

$\mathrm{H}$
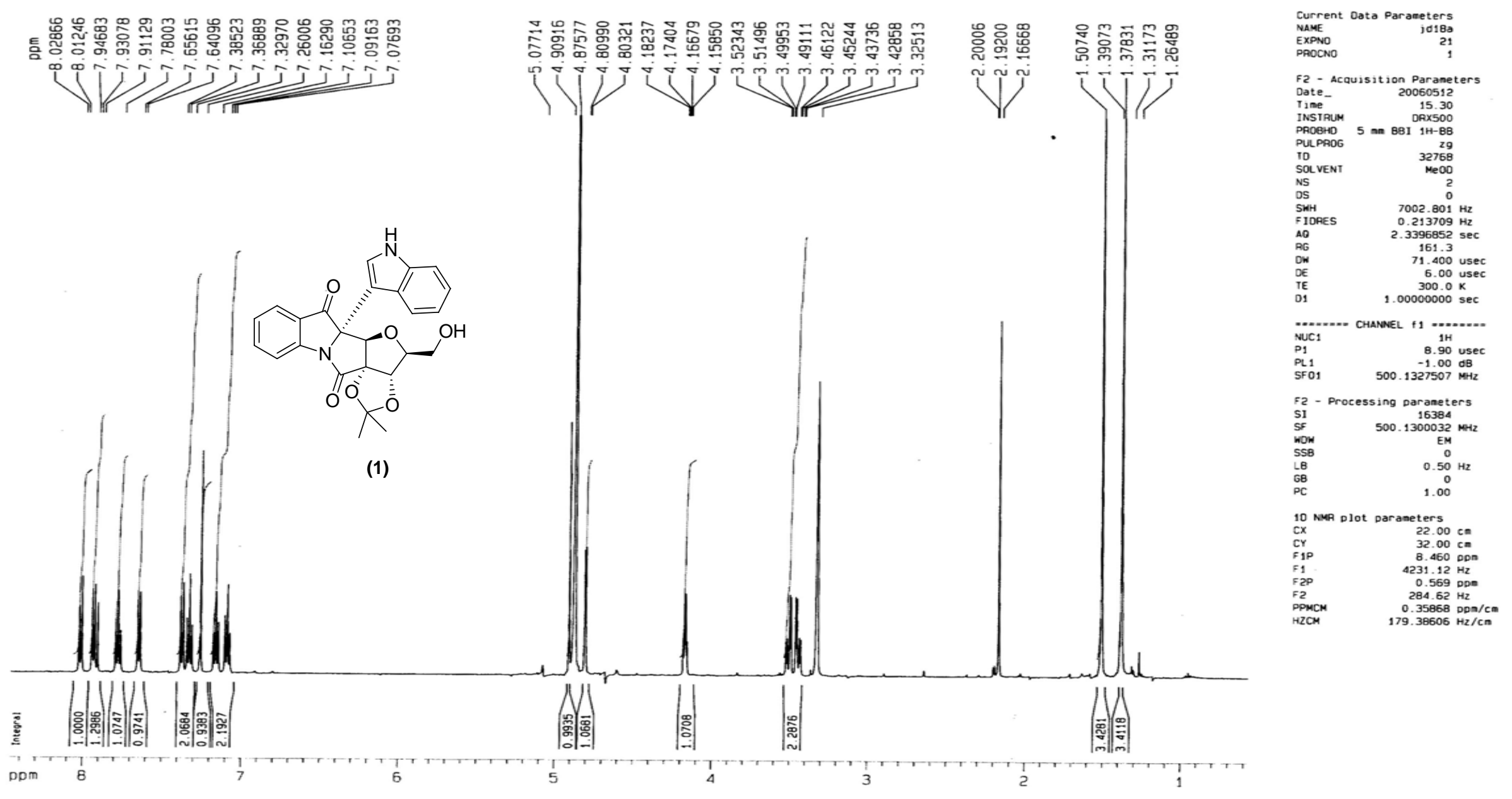
The expanded ${ }^{1} \mathrm{H}$ NMR spectrum (7.0-8.0 ppm) of compound 1

jo 18 a

$\mathrm{H}$

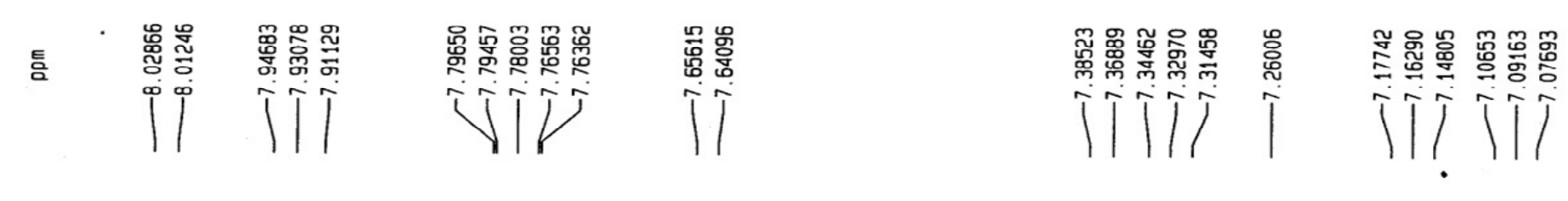

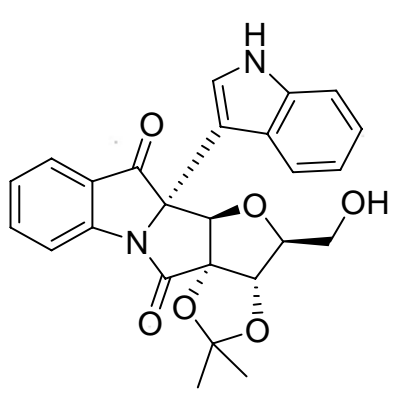

(1)

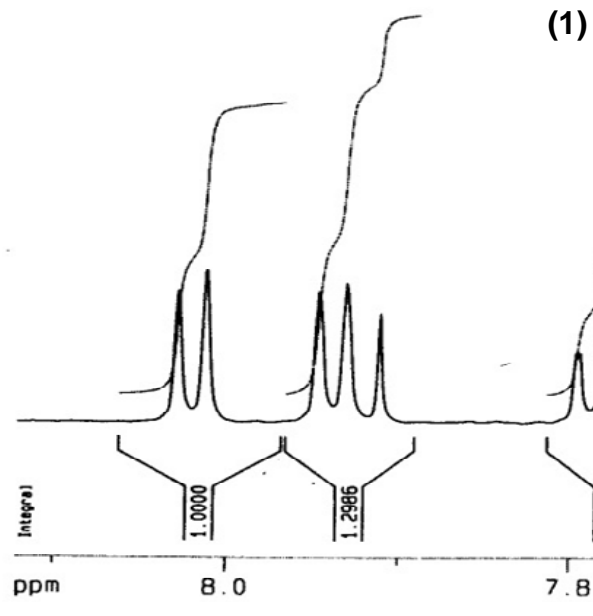

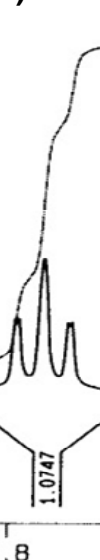

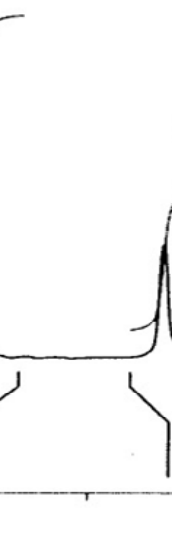

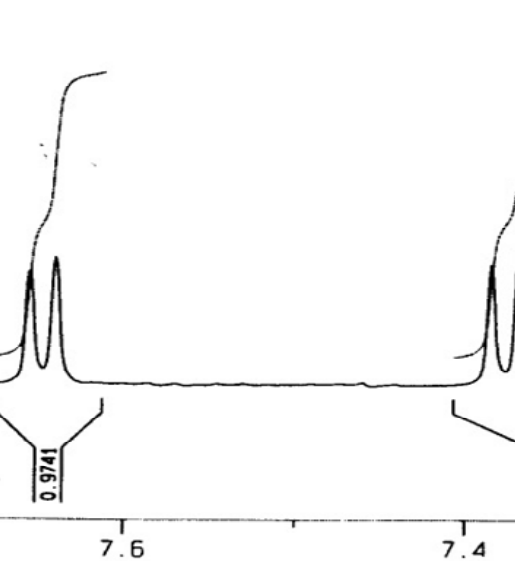

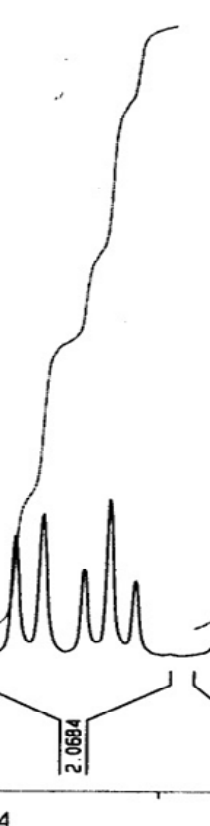

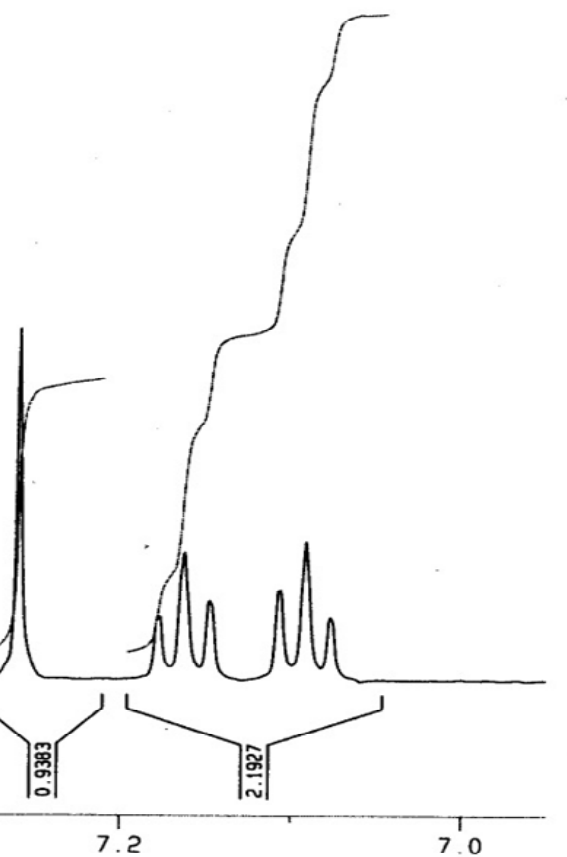

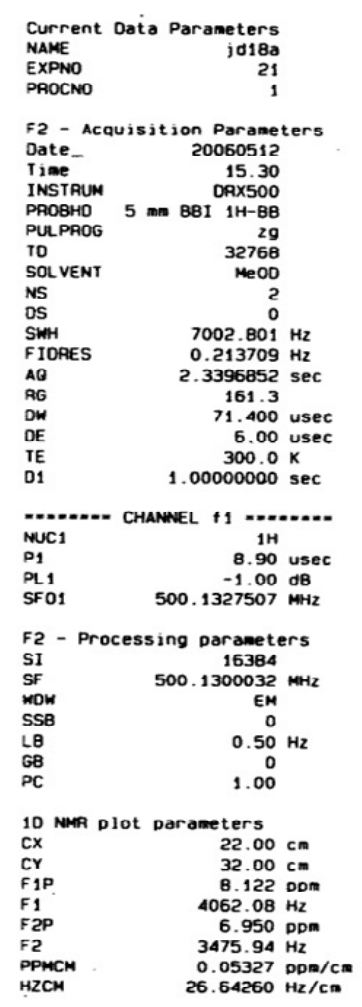


The expanded ${ }^{1} \mathrm{H}$ NMR (3.0-5.0 ppm) spectrum of compound 1

jd18a $\quad \mathrm{H}$
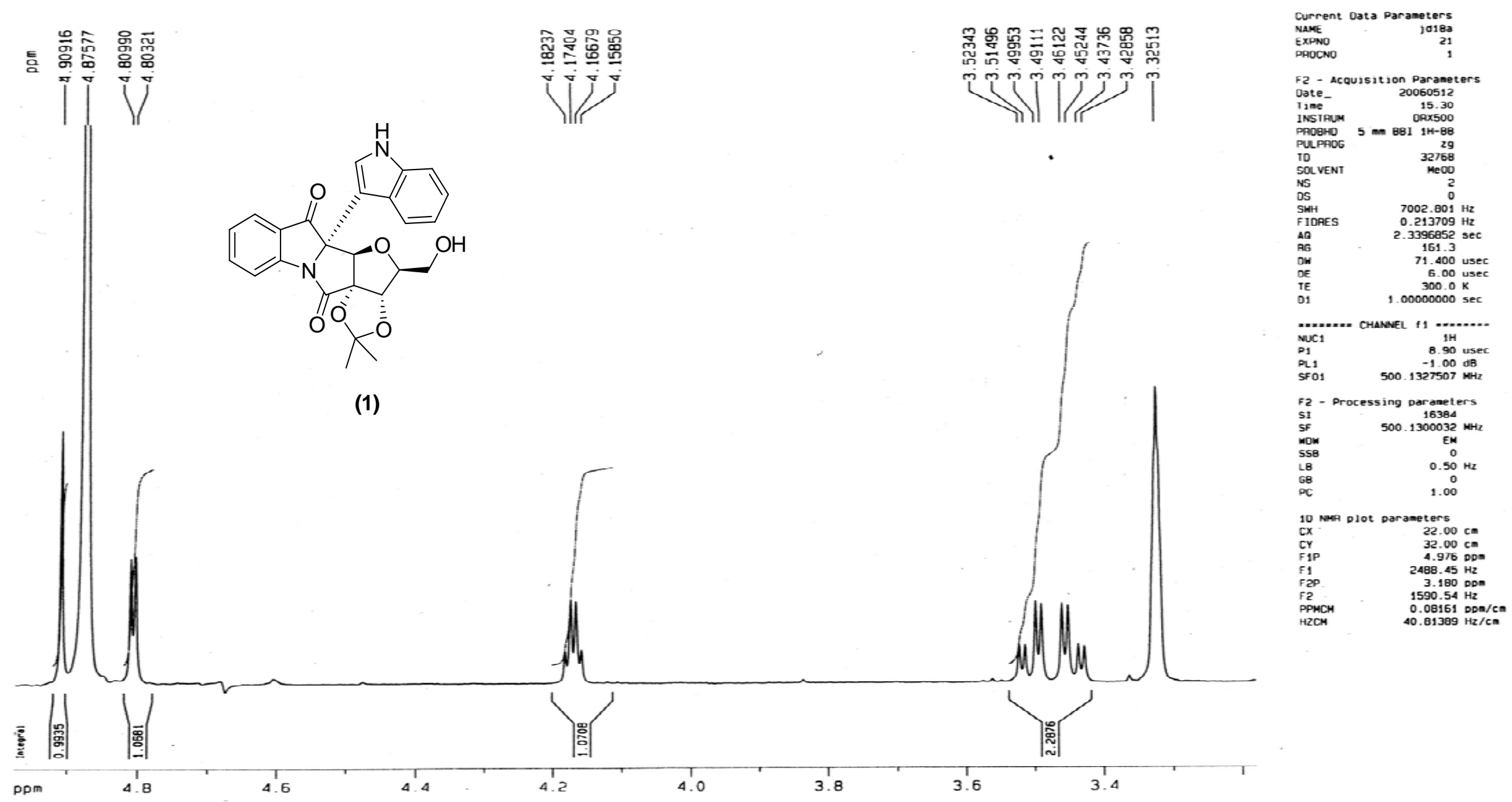


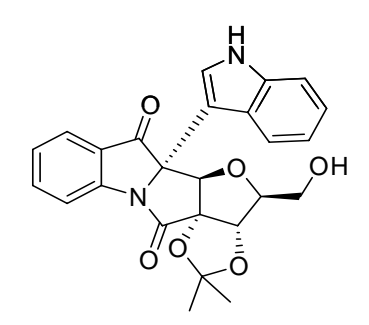

(1)

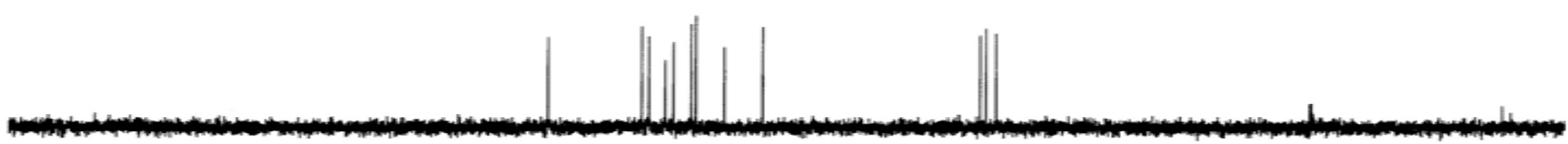

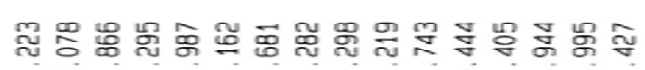

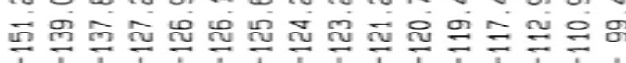

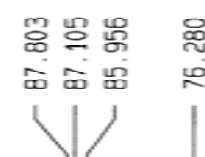

镸兽

$\frac{\sqrt[9]{5}}{\bar{\Xi}}$

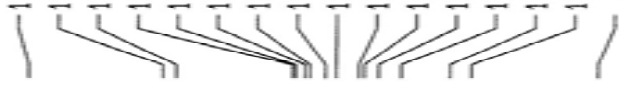

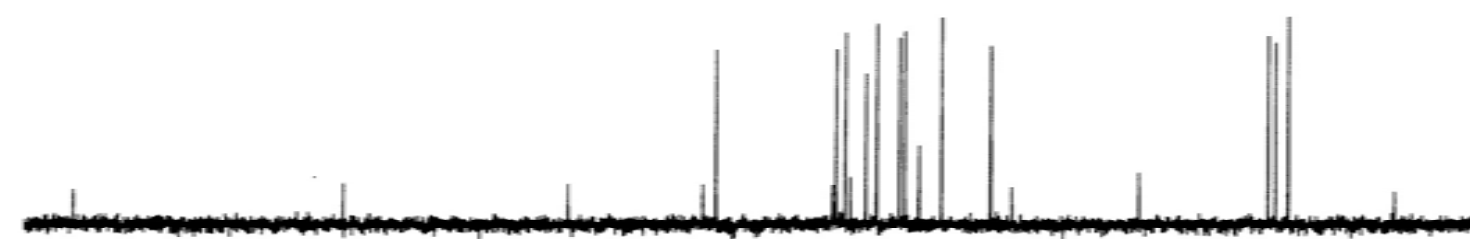

$\mathrm{ppm}$

180
160"
140
120
${ }_{100}^{T}$
BO

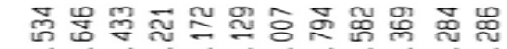

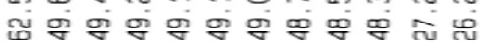

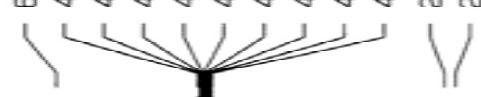

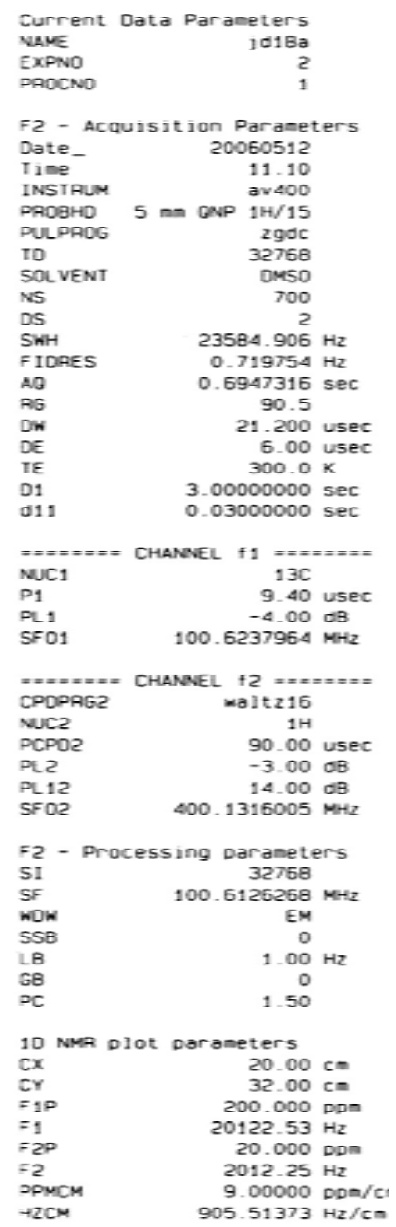




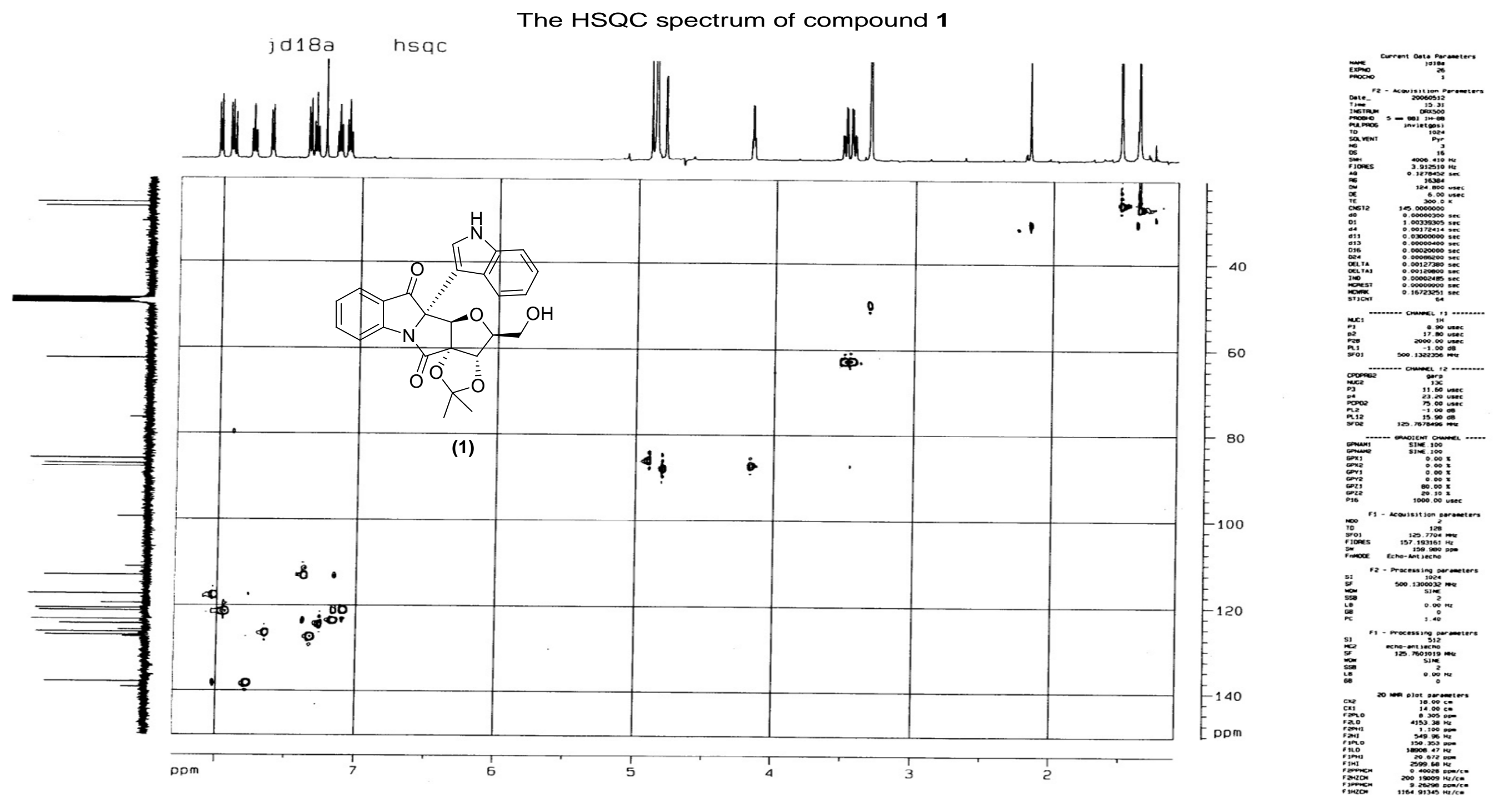


The expanded HSQC spectrum of compound 1
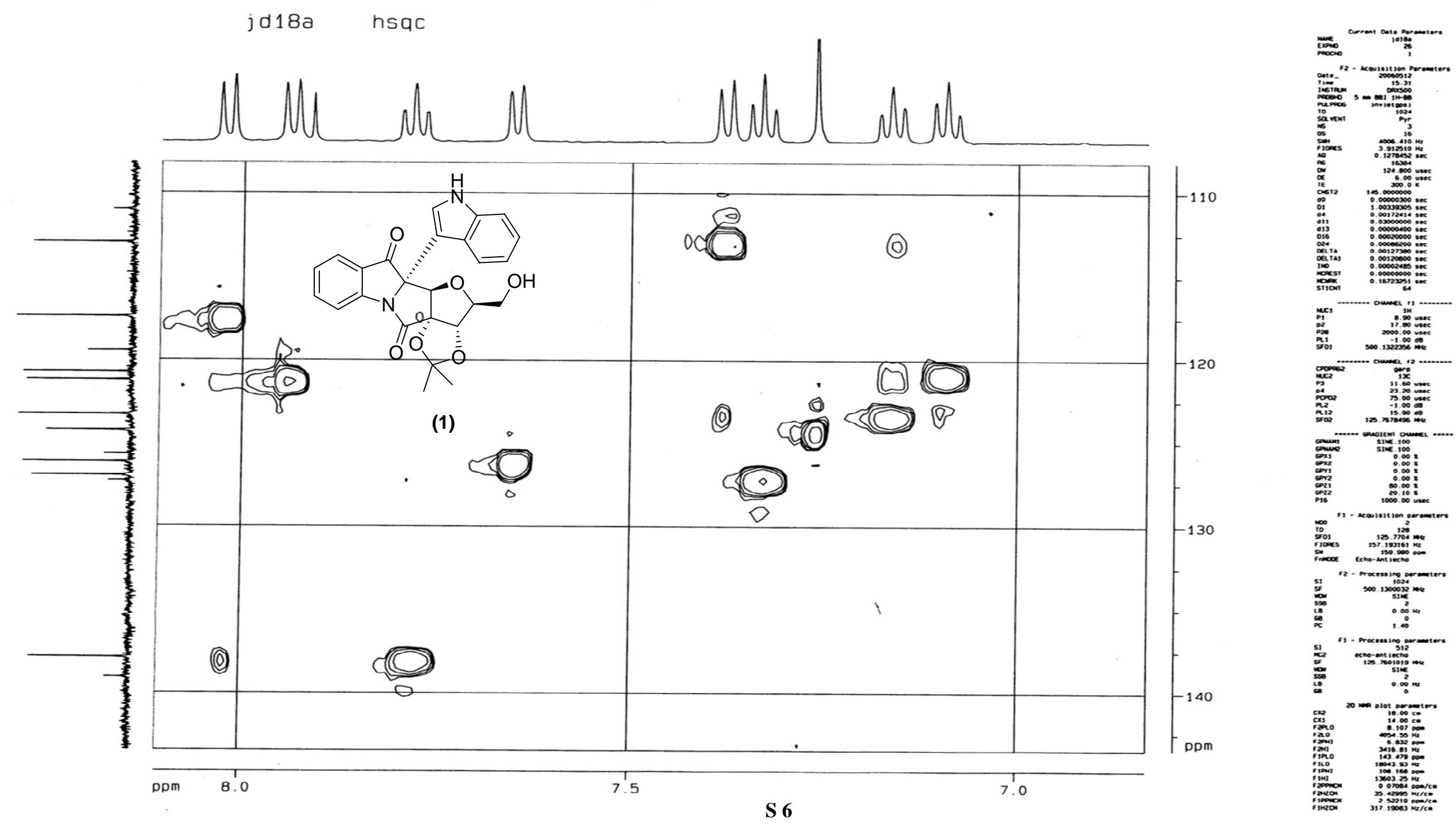
The HMBC spectrum of compound 1
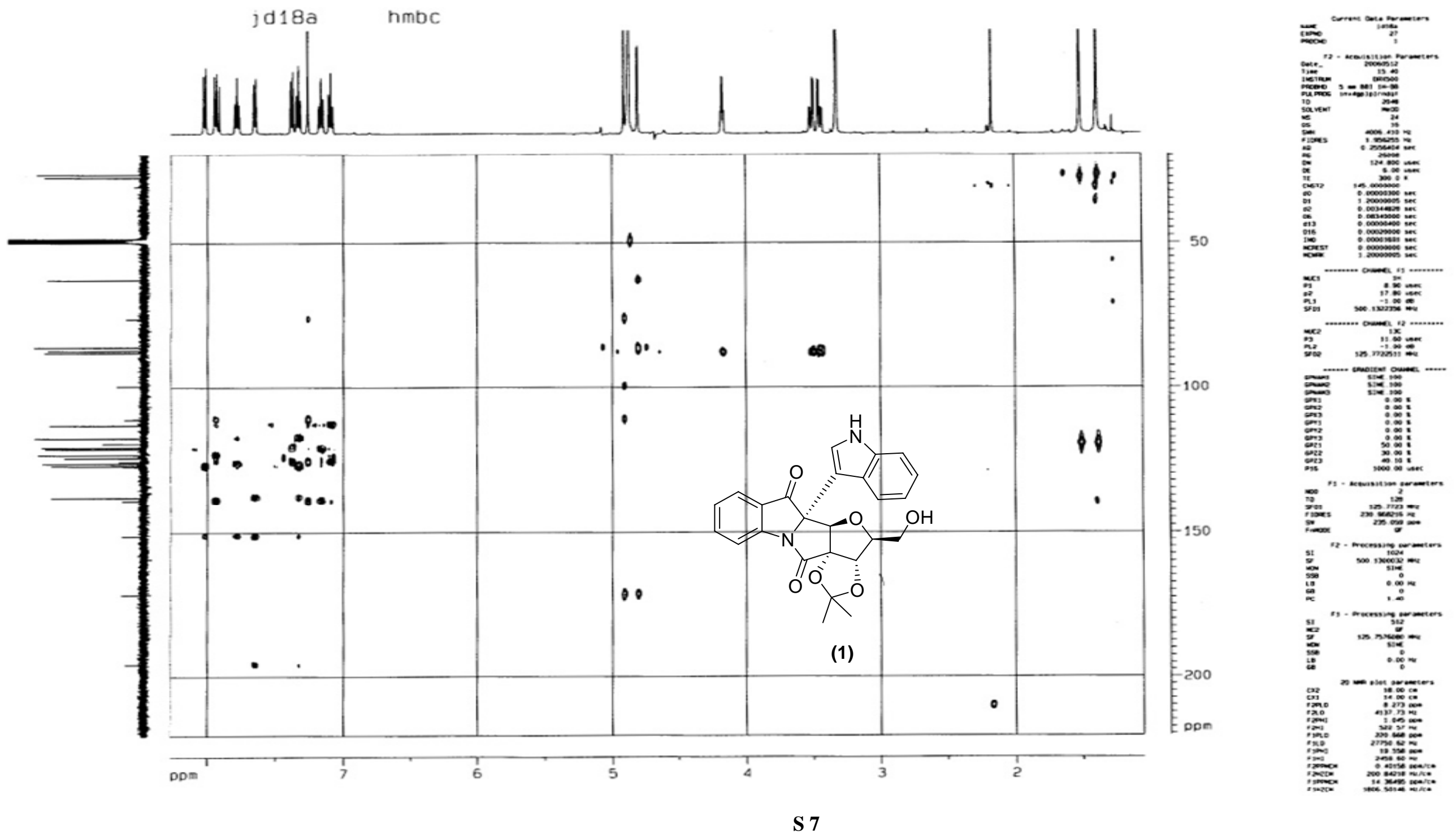
The expanded (3.0-5.0 ppm) HMBC spectrum of compound 1
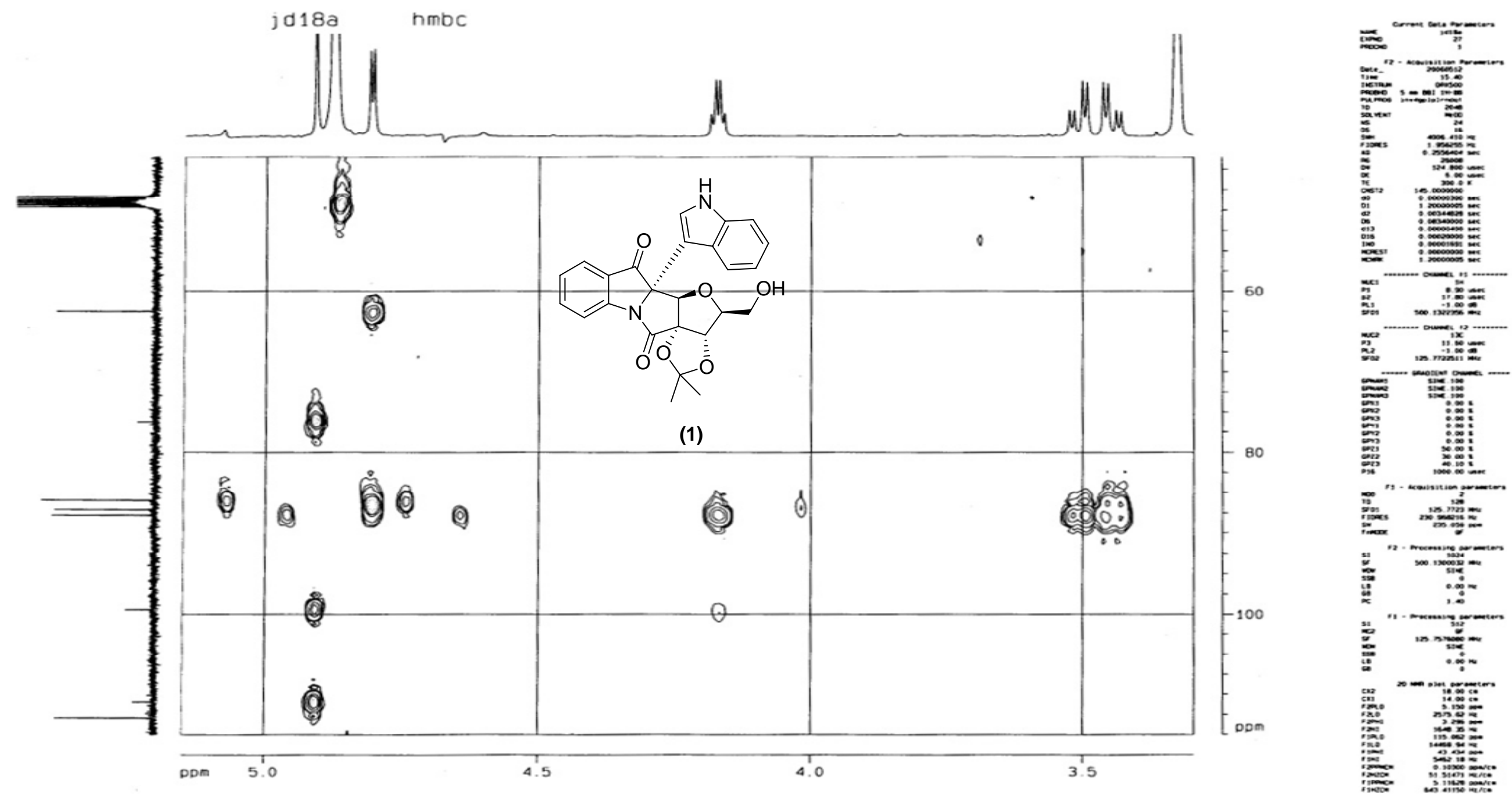
The expanded (7.0-9.0 ppm) HMBC spectrum of compound 1
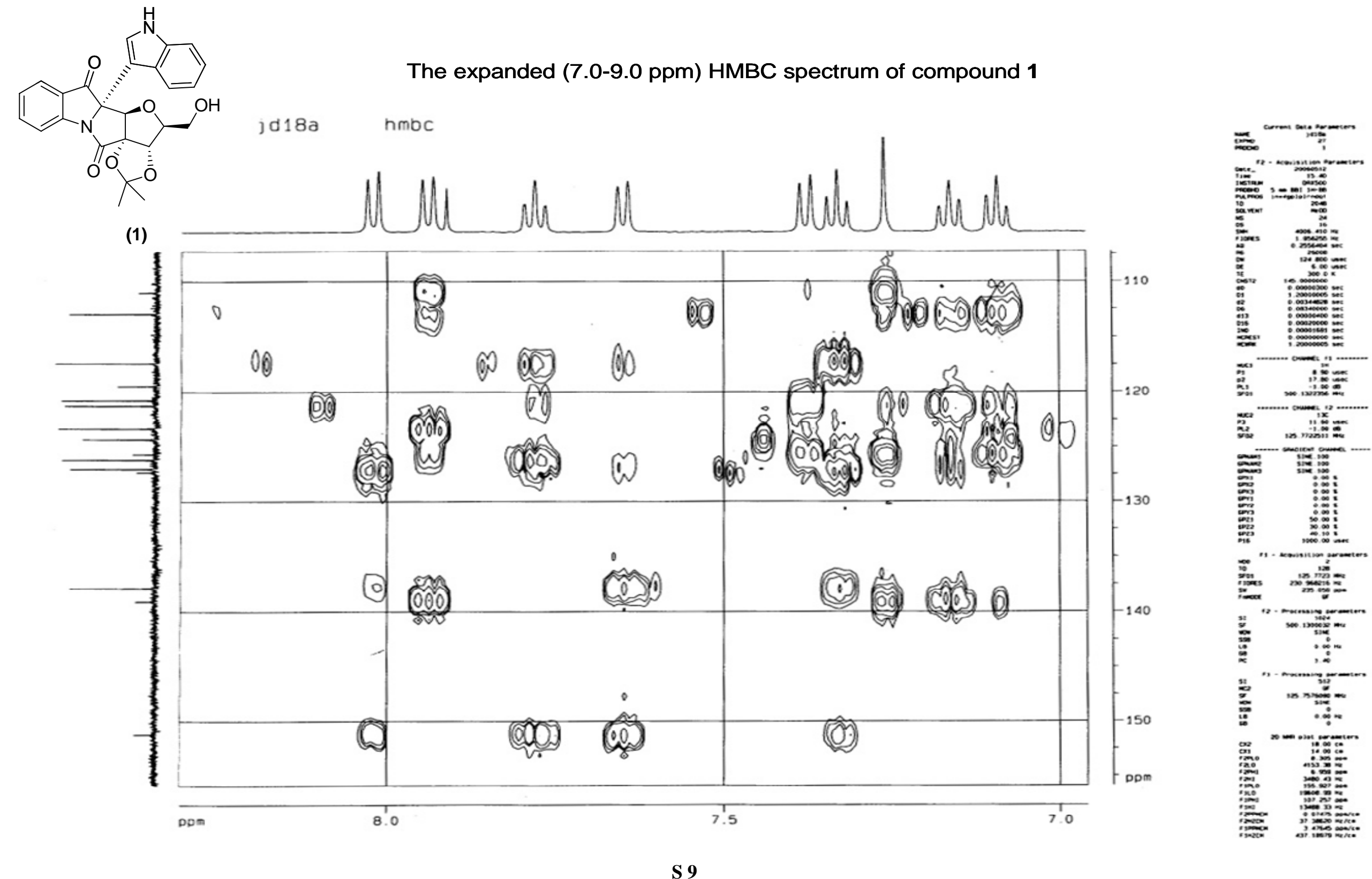


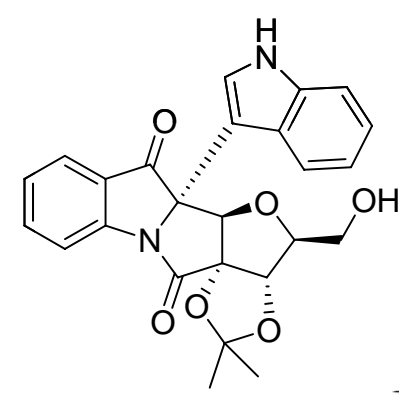

The ${ }^{1} \mathrm{H}-{ }^{1} \mathrm{H}$ COSY spectrum of compound 1
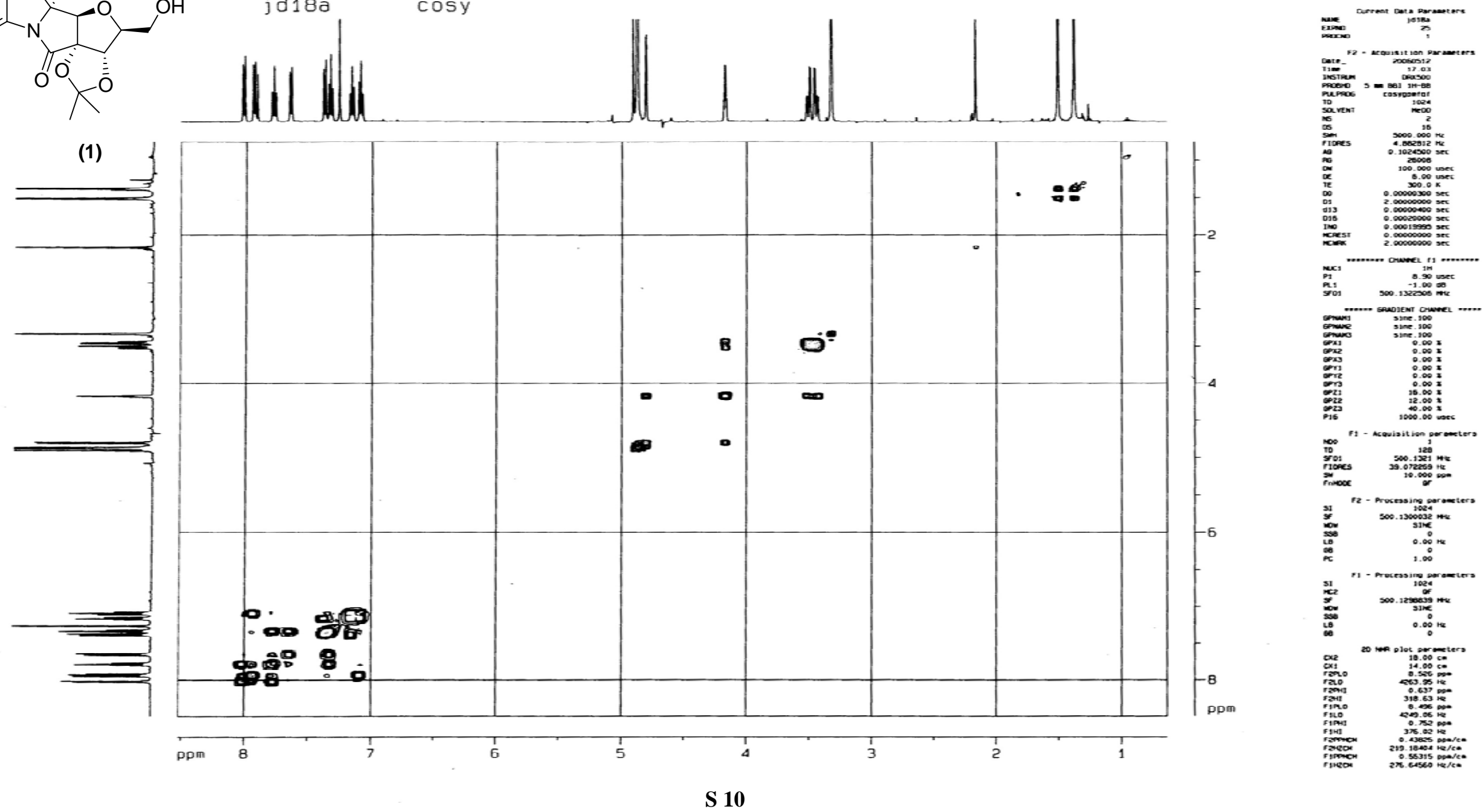

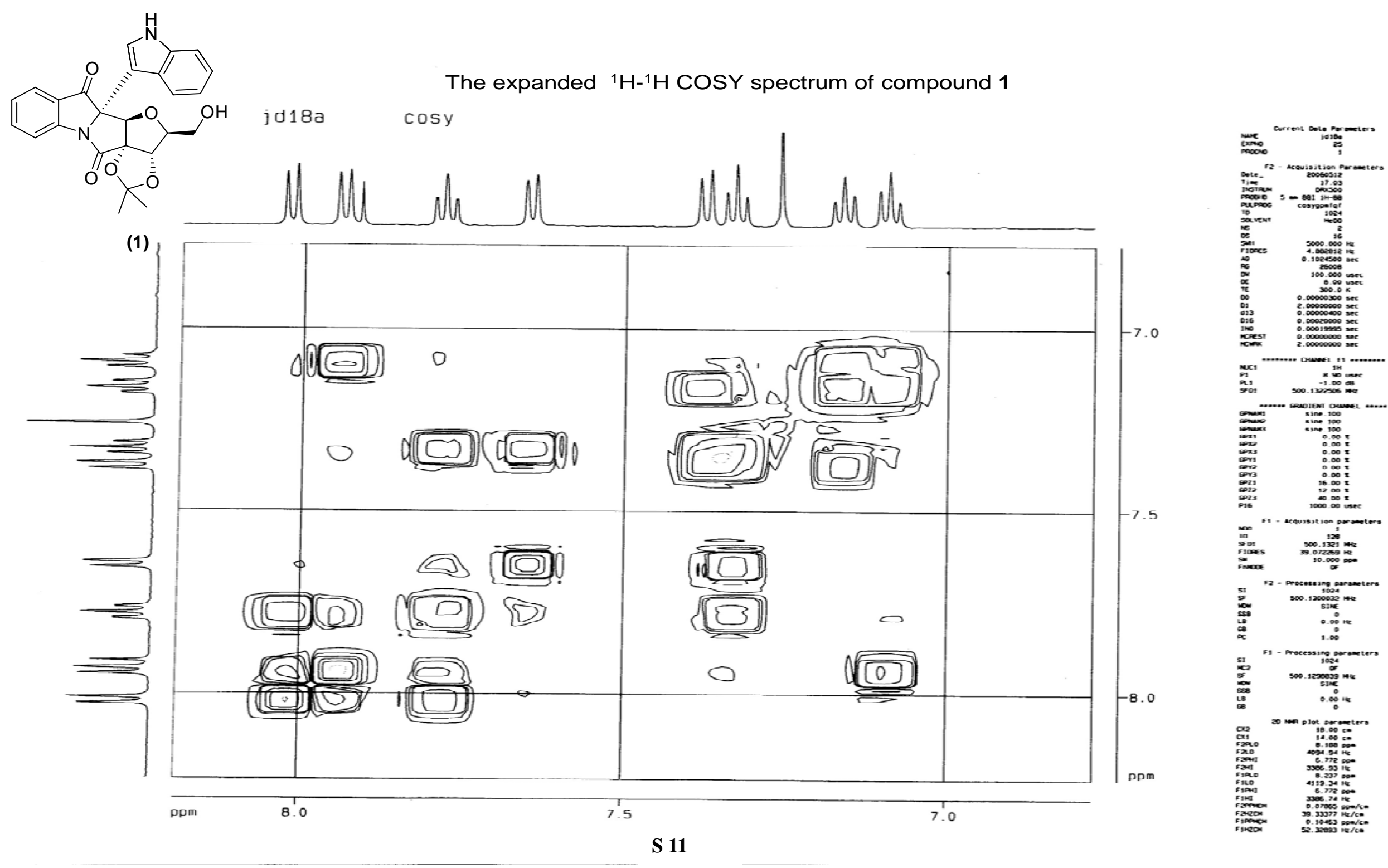
The IR(KBr) spectrum of compound 1

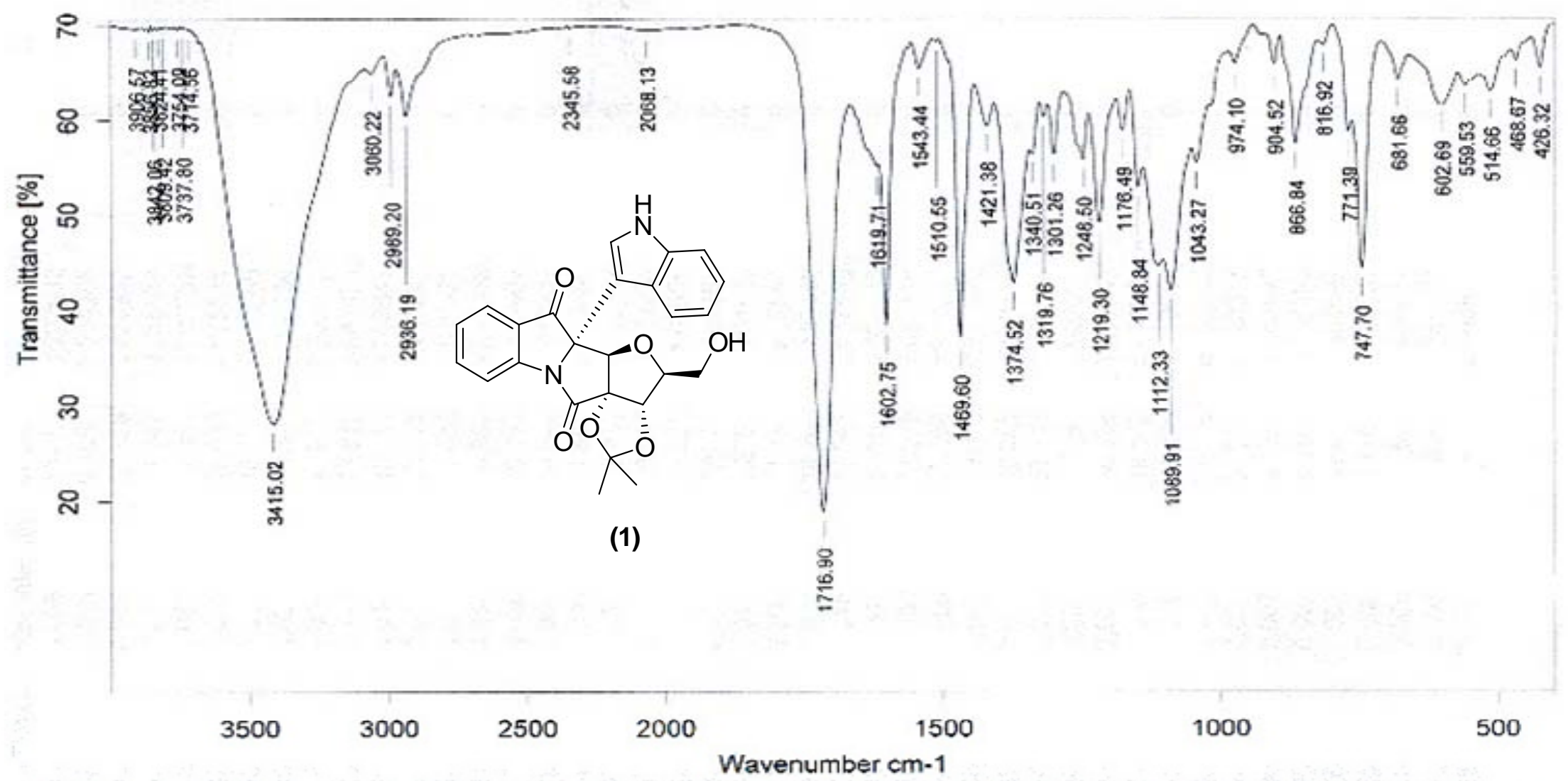

\begin{tabular}{|c|c|c|c|c|}
\hline \multicolumn{2}{|l|}{ Sample : JD-18 } & Frequency Range : 399.271 - 3996.57 & \multicolumn{2}{|c|}{ Measured on : $02 / 02 / 2007$} \\
\hline 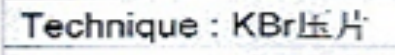 & Resolution : 4 & Instrument: Tens & 27 & Sample Scans : 16 \\
\hline Customer : 070202IR & Zerofilling : 2 & Acquisition: Douk & Sided,For & \\
\hline
\end{tabular}




\section{FABMS of compound 1}

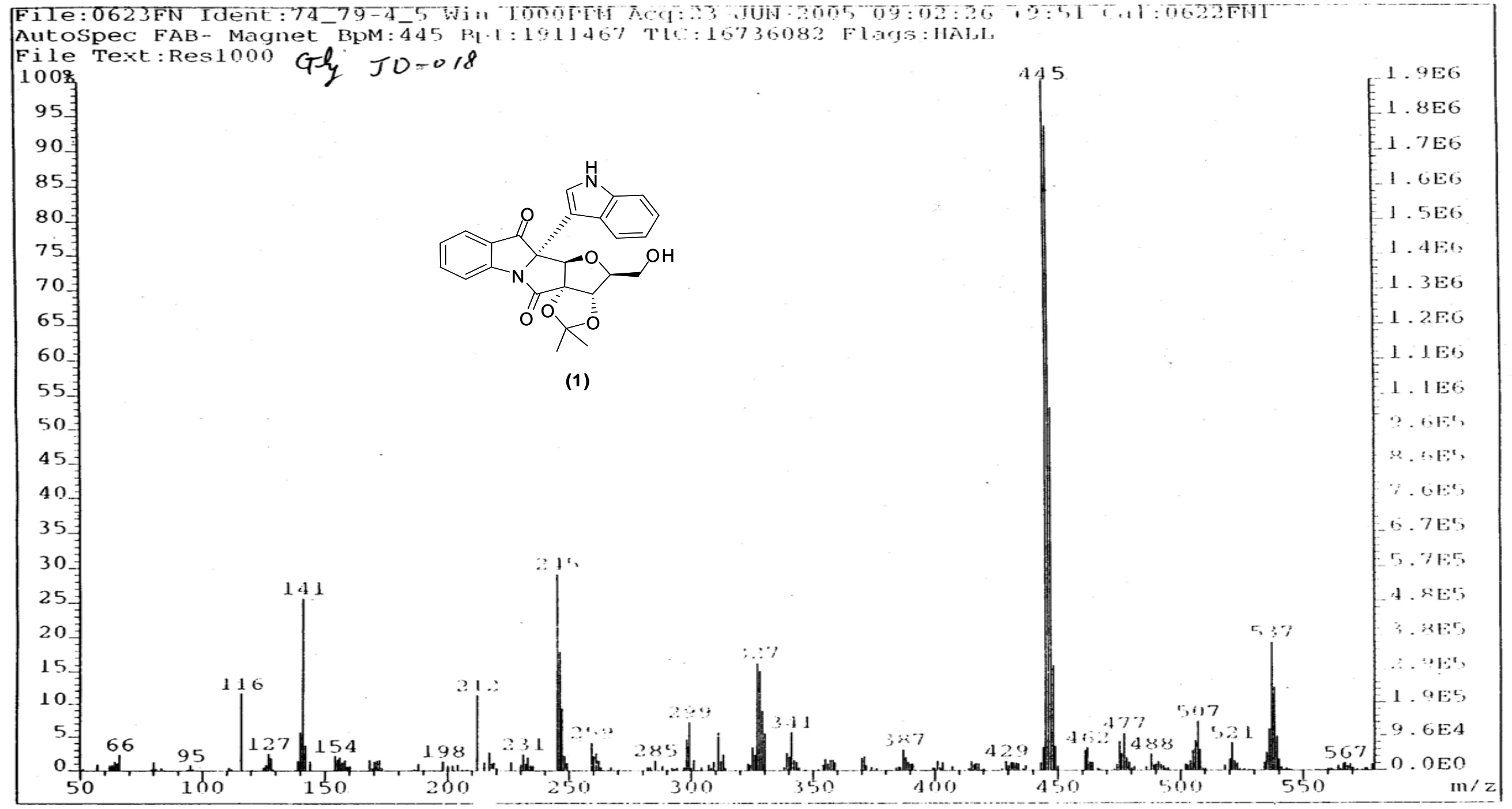


Positive HRESIMS (compound 1)

Acq. Date: Wednesday, May 17, 2006

Sample Name: O60517ESINA TD-18
Acq. Time: $09: 25$

Sample Comment:
Scan Mode: zero width

Max. 654.1 counts

-TOF MS: 3.634 to $3.750 \mathrm{~min}$ from Sample 2 (060517ESINA TD-18) of 060517ESINA TD-18.wiff $\mathrm{a}=3.55916397317915560 \mathrm{e}-004, \mathrm{t} 0=9.76406851490173720 \mathrm{e}+001$

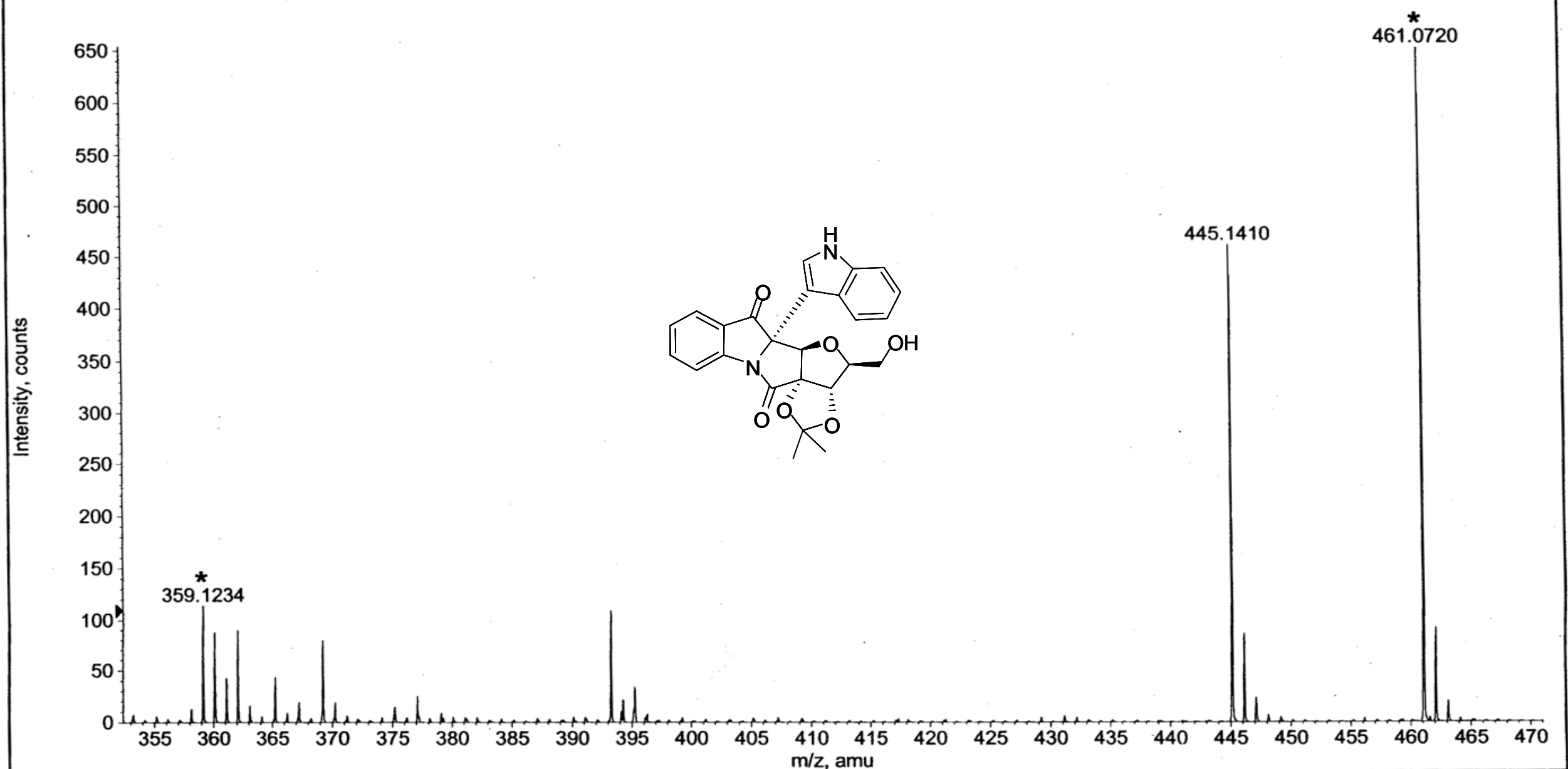


Positive HRESIMS (compound 1 )

Acq. Date: Wednesday, May 17, 2006

Sample Name: 060517ESINA TD-18

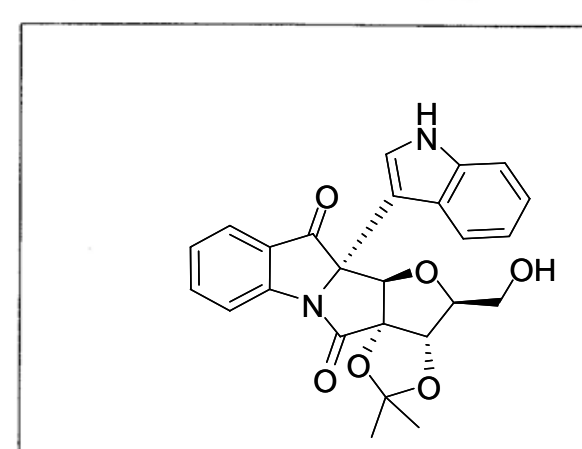

(1)
Acq. Time: $09: 25$

Sample Comment:
Scan Mode: Zero width

\section{Elemental composition calculator}

Target $\mathrm{m} / \mathrm{z}: \quad+445.1410 \quad$ amu

Tolerance: $\quad+10.0000 \quad \mathrm{ppm}$

Result type: Elemental

Max num of results: 1000

Min DBE: $\quad-5.0000 \quad$ Max DBE: +60.0000

Electron state: OddAndEven

Num of charges: 0

Add water: N/A

Add proton: N/A

File Name: 060517ESINA TD-18.wiff

\begin{tabular}{|c|c|c|c|}
\hline & Elements & Min Number & Max Number \\
\hline 1 & $\mathrm{Br}$ & 0 & 0 \\
\hline 2 & C & 0 & 80 \\
\hline 3 & $\mathrm{Cl}$ & 0 & 0 \\
\hline 4 & $F$ & 0 & 0 \\
\hline 5 & $\mathrm{H}$ & 0 & 150 \\
\hline 6 & I & 0 & 0 \\
\hline 7 & $\mathrm{~K}$ & 0 & 0 \\
\hline 8 & $\mathbf{N}$ & 0 & 4 \\
\hline 9 & $\mathrm{Na}$ & 0 & 0 \\
\hline 10 & 0 & 0 & 20 \\
\hline
\end{tabular}


Positive HRESIMS (compound 1)

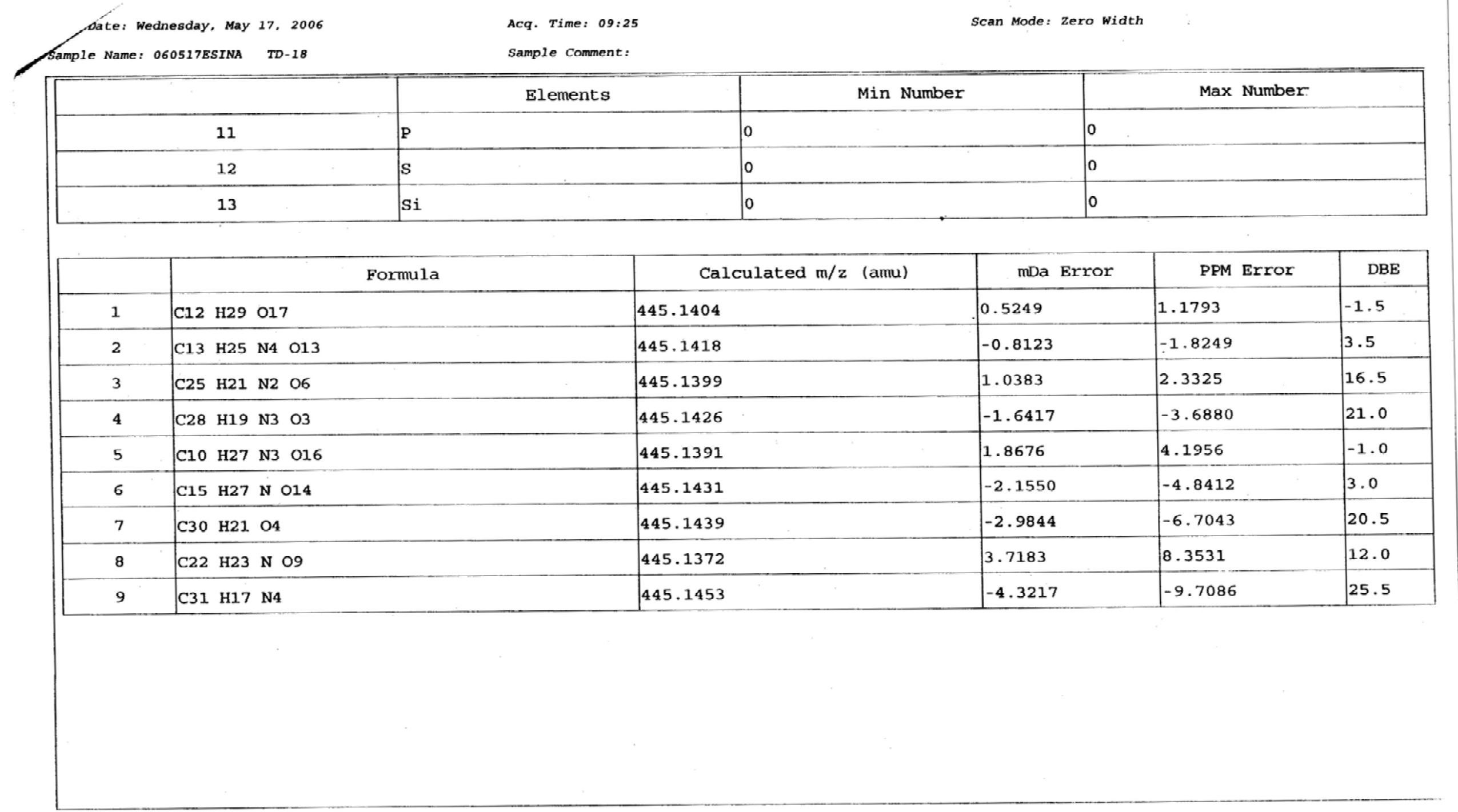


The ${ }^{1} \mathrm{H}$ NMR spectrum of compound 2

$$
\text { jd18c } \mathrm{H}
$$

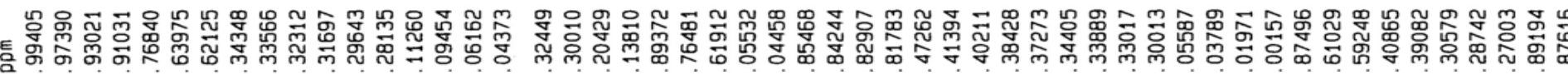
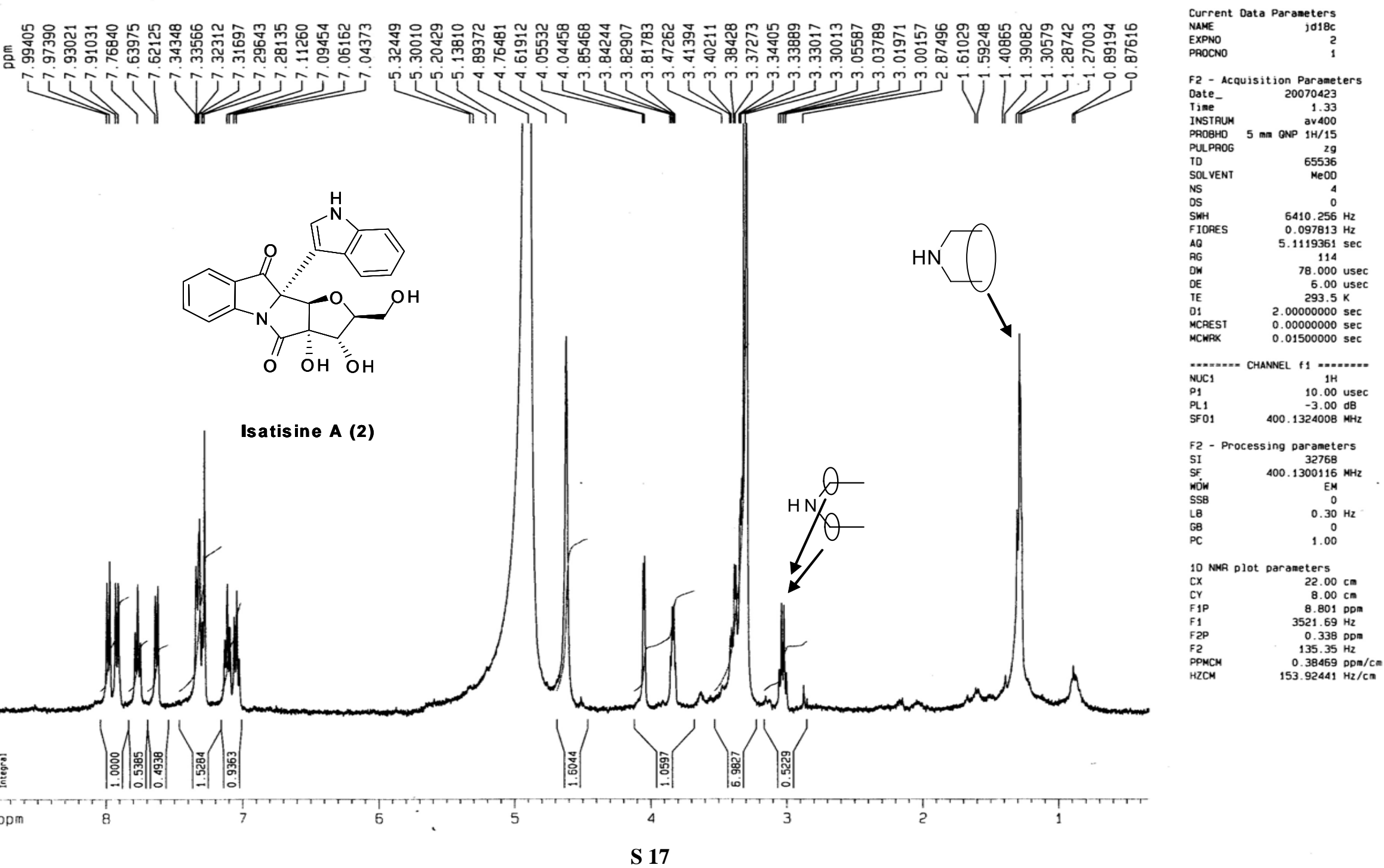


$$
\text { jd18c H }
$$

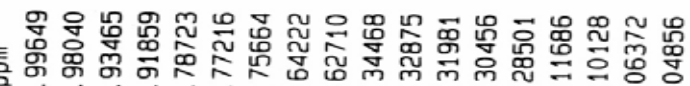

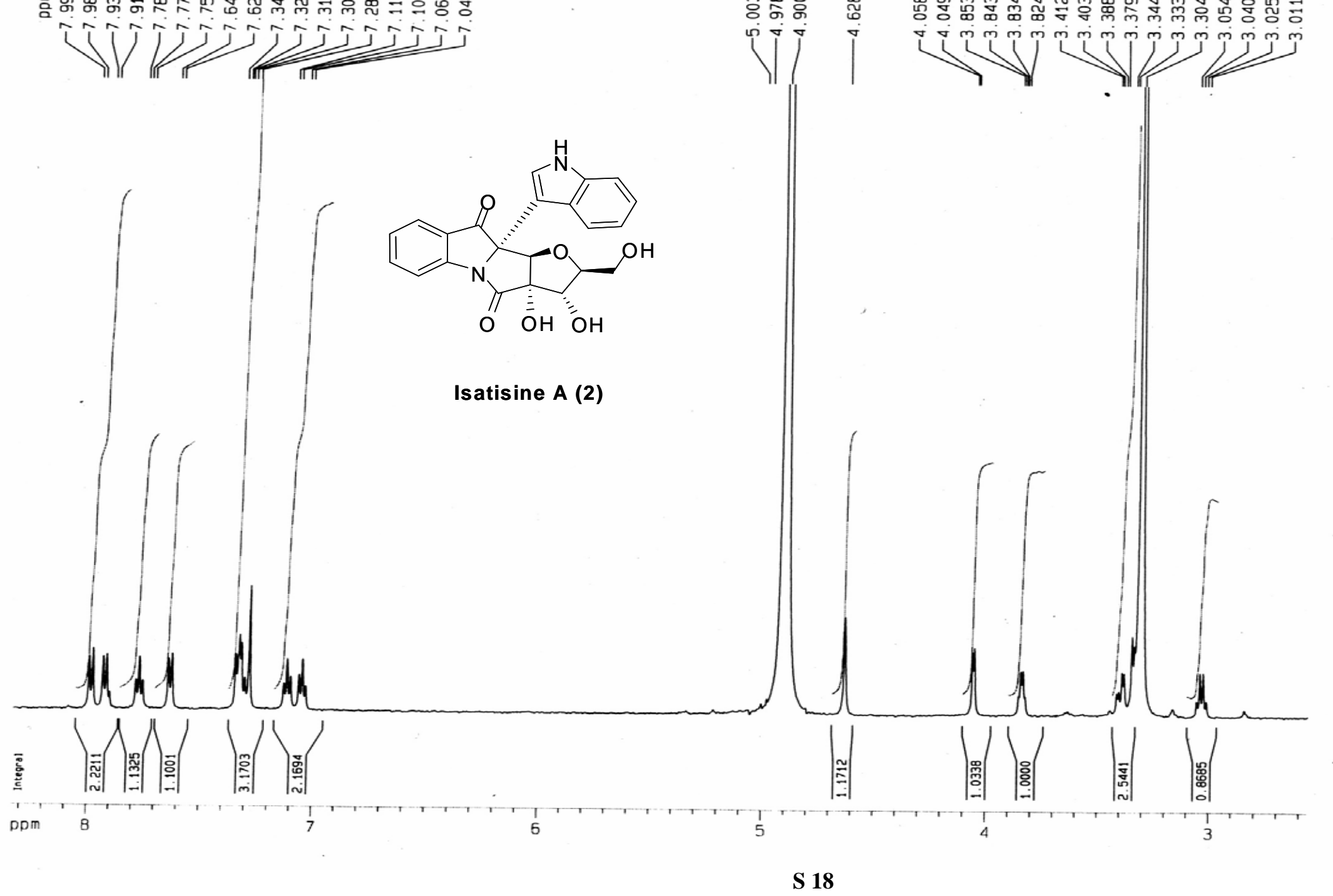

Current Data Parameters
NAME

$\begin{array}{lr}\text { NAME } & \text { Jd18C } \\ \text { EXPNO } & 21\end{array}$

F2 - Acquisition Parameter

INSTRUM

PULPPOG $5 \mathrm{~mm}$ BBI $\begin{array}{r}1 \mathrm{H}-\mathrm{BB} \\ 29\end{array}$

SOLVENT

NS
DS

$\begin{array}{lc} & 1 \\ \text { SWH } & 0 \\ & 7002.801 \\ \mathrm{~Hz}\end{array}$

FO $\quad 2.213709 \mathrm{~Hz}$

$\begin{array}{ll}A G & 181 \\ O W & 71.400 \text { usec } \\ O E & 5.00 \text { usec }\end{array}$

$\begin{array}{ll}\text { OE } & 6.00 \text { use } \\ \text { TE } & 300.0 \mathrm{~K} \\ 01 & 1.000000 \mathrm{sec}\end{array}$

......... CHANNEL +1 ........

$\begin{array}{ll}\text { NuC1 } & 1 \mathrm{H} \\ \mathrm{P}_{1} & 9.20 \mathrm{usec}\end{array}$

$\begin{array}{lr}\text { PL1 } & -1.200 \text { usec } \\ \text { SF01 } & 500.0325001 \mathrm{MHz}\end{array}$

F2 - Processing parameters

$\begin{array}{ll}\text { SI } & 16384 \\ \text { SF } & 500.0300110 \\ \text { MHZ }\end{array}$

NOW

GB

10 NMR plot para

CX
$\mathrm{CX}$

$\begin{array}{lr}\mathrm{Cr} & 130.00 \mathrm{~cm} \\ \mathrm{~F} 1 \mathrm{P} & 8.333 \mathrm{DDm}\end{array}$

$\begin{array}{ll}\text { F1 } & 4166.90 \mathrm{~Hz} \\ \text { F2P } & 2.561 .0 \mathrm{~Hz}\end{array}$

$1280.33 \mathrm{~Hz}$

PPMCM $\quad 0.26240 \mathrm{DDm} / \mathrm{cm}$ 
The FABMS of compound 2

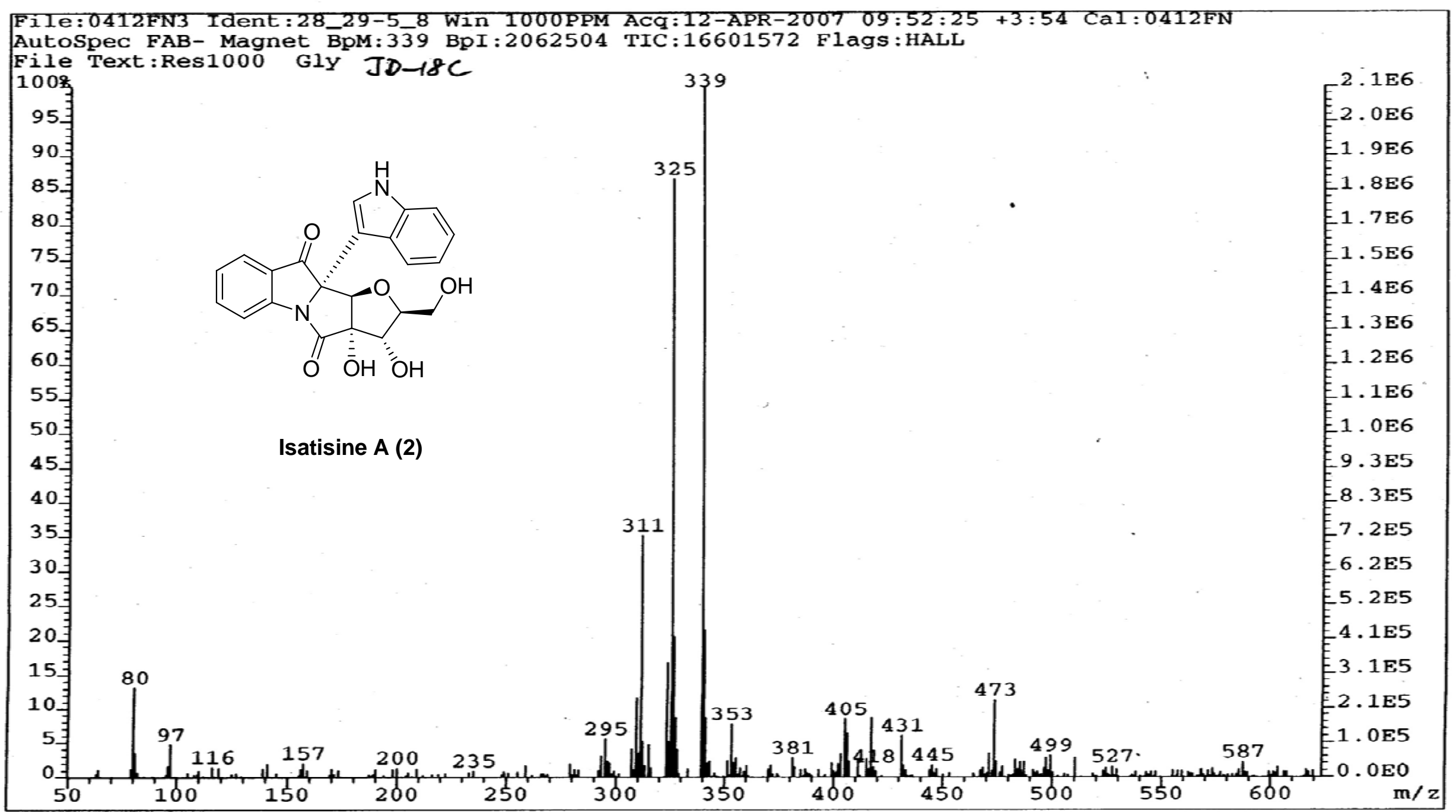




\section{Supporting information (2)}

\section{General.}

Melting point: XRC-1 apparatus (Sichuan University, Sichuan, P. R. China); HPLC ( Shimadzu LC-2010A HPLC system, Japan); Optical rotation was carried out on a Jasco DIP-370 digital polarimeter. UV spectrum was obtained from a Shimadzu UV-2401PC spectrometer. IR spectrum was measured in a Bio-Rad FTS-135 spectrometer with $\mathrm{KBr}$ pellets. HR-ESIMS was recorded on a API QSTAR PULSAP LC-Q-TOF spectrometer. 1D and 2D NMR spectra were measured on a Bruker DRX-500 instrument with residual $\mathrm{CD}_{3} \mathrm{OD}\left(\delta_{\mathrm{H}} 3.30\right.$ and $\left.\delta_{\mathrm{C}} 49.0 \mathrm{ppm}\right)$ as internal standard. Column chromatography was performed on silica gel (200-300 mesh; Qingdao Meigao Group Co., Qingdao, P.R. China), silica gel H (10-40 $\mu$; Qingdao Meigao Group Co.), and Sephadex LH-20 (40-70 $\mu$, Amersham Biotech AB, Uppsala, Sweden). Precoated silica gel GF254 (Qingdao Meigao Group Co., Qingdao, P. R. China) was used for TLC. Fractions were monitored by TLC, and spots were visualized by heating silica gel plates sprayed with $5 \%$ $\mathrm{H}_{2} \mathrm{SO}_{4}$ in $\mathrm{EtOH}$.

\section{Experiment}

To trace the origin of $\mathbf{1}$, a hydrolysis experiment was conduct to supply compound $\mathbf{2}$ (isatisine A). Detailed experiment was depict as follows: Compound 1 (1.9 mg) was dissolved in $3 \mathrm{ml}$ of methanol and then $0.05 \mathrm{ml}$ hydrochloric acid $(5 \%)$ was added. The mixture was allowed to stand for $24 \mathrm{hrs}$ at room temperature with stirring. The hydrolysate was diluted 2-fold with $\mathrm{H}_{2} \mathrm{O}$ and extracted with EtOAc. The extract was concentrated in vacuum to give a residue $(1.5 \mathrm{mg})$, which was purified by silica gel CC eluting with $\mathrm{CHCl}_{3}-\mathrm{MeOH}(9: 1)$ to yield compound 2 (1.1 mg).

In order to check the origin of compound 1, $1.8 \mathrm{~g}$ of EtOAc extract was fractionated by silica gel column ( $20 \mathrm{~g}$, 200-300 mesh) with gradient elution (petroleum ether/EtOAc, from 9:1 to 1:9) to obtain fractions 1-7. With the authentic sample compound 1 and isatisine A (2) as references, Frs.1-7 was analyzed respectively by TLC (petroleum ether: EtOAc $=6: 4, \mathrm{Rf}=0.5$ for compound 1, $\mathrm{CHCl}_{3}: \mathrm{MeOH}=9: 1, \mathrm{Rf}=0.5$ for isatisine A). Results revealed that compound $\mathbf{1}$ could not be found in any fraction, and isatisine A (2) was detected only in fraction 5. To further comfirm this result, fraction 5 was analyzed

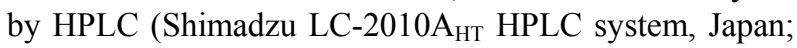
column: Zorbax $\mathrm{SB} \mathrm{C}_{18}$, Agilent, $4.6 \mathrm{~mm} \times 25 \mathrm{~cm}$, U.S.A.; detected at $254 \mathrm{~nm}$; mobile phase: $\mathrm{MeOH} / \mathrm{H}_{2} \mathrm{O}$ 50:50; flow rate: $1 \mathrm{ml} / \mathrm{min}$ ). As shown in figure 4 , isatisine A (2) could be detected in fraction 5 with a retention time at about $7.3 \mathrm{~min}$, but compound $\mathbf{1}$ could not be detected with a retention time at about $20.9 \mathrm{~min}$.
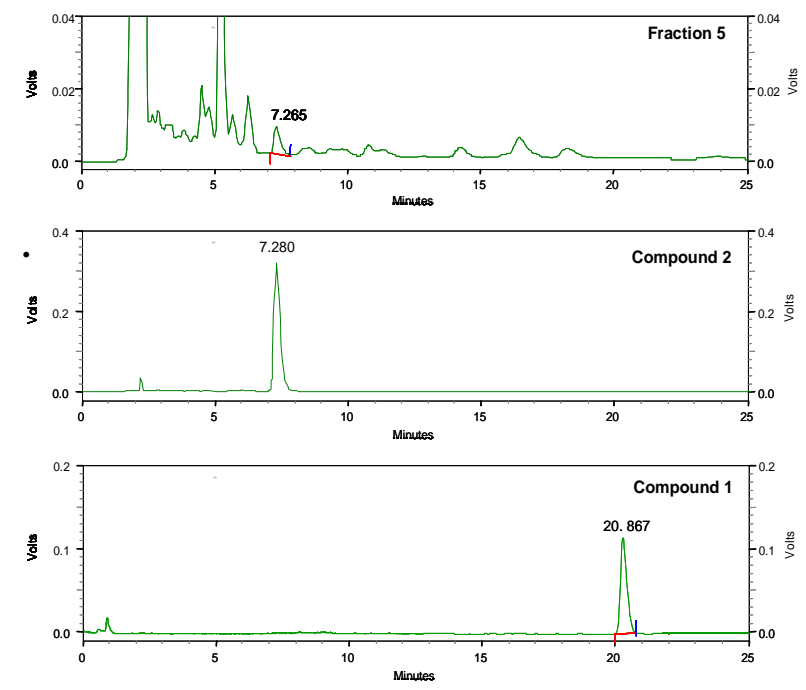

Figure 4. HPLC chromatograms of fraction 5, compound $\mathbf{2}$ and compound $\mathbf{1 .}$ 
The X-ray diffraction of compound 1

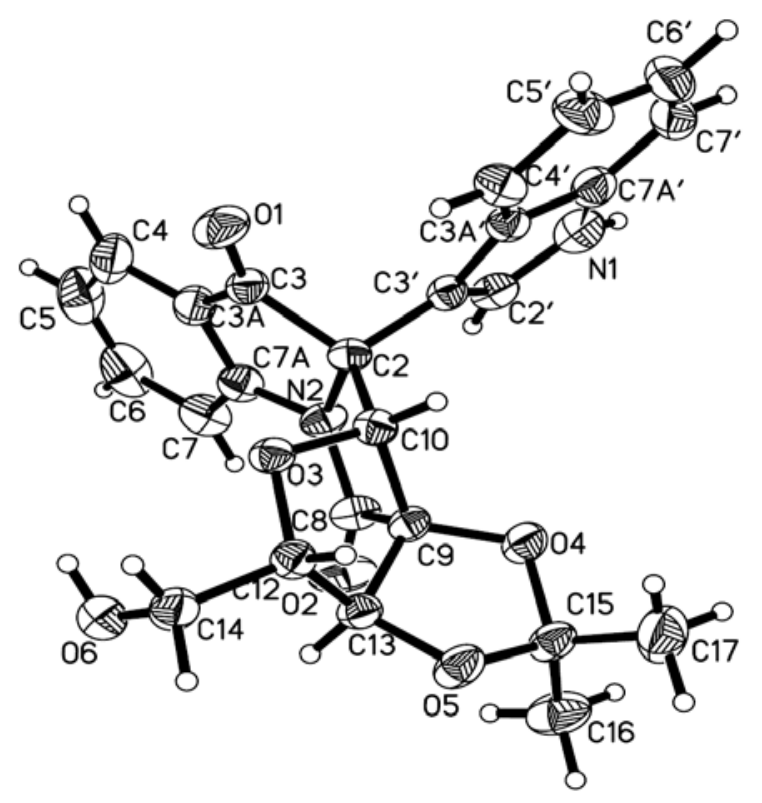

